\title{
Identification of Transcription Factors ZmMYB111 and ZmMYB148 Involved in Phenylpropanoid Metabolism
}

\author{
Junjie Zhang ${ }^{1+}$, Shuangshuang Zhang ${ }^{2+}$, Hui Li2t, Hai Du ${ }^{3}$, Huanhuan Huang ${ }^{2}$, \\ Yangping $\mathrm{Li}^{2}$, Yufeng Hu${ }^{2}$, Hanmei Liu', Yinghong Liu${ }^{4}$, Guowu Yu${ }^{1}$ and Yubi Huang ${ }^{2 *}$ \\ ${ }^{1}$ College of Life Science, Sichuan Agricultural University, Chengdu, China, ${ }^{2}$ College of Agronomy, Sichuan Agricultural \\ University, Chengdu, China, ${ }^{3}$ College of Agronomy and Biotechnology, Southwest University, Chongqing, China, ${ }^{4}$ Maize \\ Research Institute, Sichuan Agricultural University, Chengdu, China
}

\section{OPEN ACCESS}

Edited by:

Sergio Lanteri,

Dipartimento di Scienze Agrarie -

University of Turin, Italy

Reviewed by:

Ze-Chun Yuan,

University of Western Ontario, Canada

Andrea Moglia,

University of Turin, Italy

${ }^{*}$ Correspondence:

Yubi Huang

yubihuang@sina.com

tThese authors have contributed equally to this work.

Specialty section:

This article was submitted to Crop Science and Horticulture, a section of the journal

Frontiers in Plant Science

Received: 04 December 2015 Accepted: 28 January 2016

Published: 15 February 2016

Citation:

Zhang J, Zhang S, Li H, Du H,

Huang H, Li Y, Hu Y, Liu H, Liu Y, Yu G and Huang $Y$ (2016) Identification

of Transcription Factors ZmMYB111 and ZmMYB148 Involved

in Phenylpropanoid Metabolism.

Front. Plant Sci. 7:148.

doi: 10.3389/fpls.2016.00148
Maize is the leading crop worldwide in terms of both planting area and total yields, but environmental stresses cause significant losses in productivity. Phenylpropanoid compounds play an important role in plant stress resistance; however, the mechanism of their synthesis is not fully understood, especially in regard to the expression and regulation of key genes. Phenylalanine ammonia-lyase (PAL) is the first key enzyme involved in phenylpropanoid metabolism, and it has a significant effect on the synthesis of important phenylpropanoid compounds. According to the results of sequence alignments and functional prediction, we selected two conserved R2R3MYB transcription factors as candidate genes for the regulation of phenylpropanoid metabolism. The two candidate R2R3-MYB genes, which we named ZmMYB111 and ZmMYB148, were cloned, and then their structural characteristics and phylogenetic placement were predicted and analyzed. In addition, a series of evaluations were performed, including expression profiles, subcellular localization, transcription activation, protein-DNA interaction, and transient expression in maize endosperm. Our results indicated that both ZmMYB111 and ZmMYB148 are indeed R2R3-MYB transcription factors and that they may play a regulatory role in $\mathrm{PAL}$ gene expression.

Keywords: maize, MYB, transcription factor, phenylpropanoid metabolism, gene expression

\section{INTRODUCTION}

Maize is the leading crop worldwide in terms of both planting area and total yield (reached about 0.87 billion tons in 2012) according to the report of FAOFTAT ${ }^{1}$, and its production continues to expand in both developed and developing countries (Wang et al., 2013). It is used not only as a staple food source, but also for animal feed, biofuel, and various industrial raw materials. However, environmental stresses, such as extremes in temperature, drought, salinity, heavy metals, and oxidative stress, cause significant losses in maize productivity. To cope with these types of stress, plants are equipped with various mechanisms that enable them to survive harsh environmental conditions (Bohnert et al., 1995), including the ability to synthesize special compounds, such as phenylpropanoids (Solecka, 1997; Ramakrishna and Ravishankar, 2011). Therefore, a better

${ }^{1}$ http://faostat.fao.org/site/339/default.aspx 
understanding of the mechanism or phenylpropanoid synthesis is critical to improving the stress tolerance of maize.

Phenylpropanoid metabolism gives rise to a diverse group of compounds, which are derived from the carbon skeleton of phenylalanine and are involved in plant defense, structural support, and survival (Vogt, 2010). The phenylpropanoid pathway begins with three reactions that are respectively catalyzed by phenylalanine ammonia-lyase (PAL; EC 4.3.1.5), Cinnamate 4-hydroxylase $(\mathrm{C} 4 \mathrm{H} ; \quad \mathrm{EC}$ 1.14.13.11), and 4coumarate: CoA ligase (4CL; EC 6.2.1.12), leading to the synthesis of p-coumaroyl CoA (Fraser and Chapple, 2011), which is a common precursor for the production of many other important compounds. Lignin and flavonoid are the main phenylpropanoid compounds found. In maize, lignin is mainly distributed in the stem, root, and leaf tissues, and flavonoids are mainly located in seeds.

One important group of secondary metabolites that is derived from the phenylpropanoid pathway is the flavonoids, which is a group of polyphenolic secondary metabolites that includes the anthocyanins (red to purple pigments), flavonols (colorless to pale yellow pigments), flavonols (colorless pigments that become brown after oxidation), and proanthocyanidins (PAs) or condensed tannins (Petrussa et al., 2013). Flavonoids play a major role in plant responses to both biotic and abiotic stresses. For example, when abiotic stressors (such as phosphate, nitrogen, light, temperature, UV, or drought) cause the accumulation of reactive oxygen species (ROS), flavonoids are able to effectively scavenge ROS, thus preventing oxidative damage (Nakabayashi et al., 2014).

Another important group of phenylpropanoid-derived compounds is the lignins, which is a large group of aromatic polymers that are major components of plant cell walls and that serve a variety of functions related to both structural support and plant defense (Boerjan et al., 2003). One of the major functions of lignins is protecting cell wall polysaccharides from microbial degradation (Mansfield, 2009; Vanholme et al., 2010), and the synthesis of lignins can be induced by multiple types of abiotic stress, including mineral deficiency, drought, UV-B radiation, and low temperature, as well as by biotic stressors, such as infection by fungi, bacteria, or viruses (Moura et al., 2010).

The myeloblastosis (MYB) proteins comprise one of the largest and most functionally diverse families of transcription factors in plants and are characterized by a highly conserved DNA-binding domain, the MYB domain, which is approximately 52 amino acid residues in length and is capable of forming a helixturn-helix fold with three regularly spaced tryptophan residues (Kanei-Ishii et al., 1990; Katiyar et al., 2012). The majority of plant MYB proteins belong to the R2R3-MYB subfamily (Dubos et al., 2010; Cai et al., 2012; Katiyar et al., 2012), and the R2R3MYB transcription factors play central roles in controlling plantspecific processes, including primary and secondary metabolism, cell fate and identity, development, and response to both abiotic and biotic stresses (Dubos et al., 2010; Katiyar et al., 2012).

In previous studies, we performed a genome-wide survey of the R2R3-MYB gene subfamily in maize, in which 158 putative R2R3-MYBs were identified, and a comprehensive analysis that included phylogenetics, expression patterns, and determination of the proteins' structural and functional characteristics ( $\mathrm{Du}$ et al., 2012). Our results indicated that protein functions were conserved between maize MYB genes and their putative orthologs, based on homology, co-expression, and collinearity analyses (Du et al., 2012). Accordingly, we selected two conserved R2R3-MYB transcription factors as candidate genes for the regulation of phenylpropanoid metabolism. The two candidate R2R3-MYB genes, which we named ZmMYB111 and ZmMYB148, were cloned from the maize inbred line B73, and their regulatory mechanisms were determined.

\section{MATERIALS AND METHODS}

\section{Plant Materials}

Seeds for the B73 maize inbred line were provided by the College of Agronomy, Sichuan Agricultural University and were grown at the school farm in the summer of 2013, according to the local standards for maize production. When silks emerged, strict selfpollinations were performed every morning, and 25 days after pollination (DAP), roots, leaves, upper and lower stems, pith, and both male and female flowers were sampled for RNA isolation. All samples were immediately frozen in liquid nitrogen and stored at $-70^{\circ} \mathrm{C}$ until used. Fresh endosperms were harvested at 9 DAP for use as receptors for transient expression.

\section{RNA Extraction, Reverse Transcription, and DNA Extraction}

Total RNAs were extracted from the frozen samples using Trizol reagent (Invitrogen, China), and treated with gDNA Eraser (Takara, Dalian, China) to remove genomic DNA contamination. One $\mu \mathrm{g}$ of total RNA from each sample was used to produce cDNA for cloning both ZmMYB111 and ZmMYB148 and for measuring gene transcription levels via reverse transcription PCR (RT-PCR; PrimeScript ${ }^{\text {TM }}$ RT Reagent Kit; Takara, China). Genomic DNA (gDNA) was isolated from young leaves using the cetyltrimethylammonium bromide (CTAB) method (Fulton et al., 1995).

\section{Gene Cloning of ZmMYB111 and ZmMYB148 and Bioinformatics Analysis}

The cDNAs of ZmMYB111 (GRMZM2G104551) and ZmMYB148 (GRMZM2G097636) were amplified from cDNA templates from the upper stem samples using the following primer pairs: 5'-GAATTCATGGGTCGGCAGCCGT-3' (forward) and 5'-GAGCTCCTAGAACTTTGCTCCGTTTG-3' (reverse), and $5^{\prime}$-GAATTCATGGGGAAGGGCC-3' (forward) and $5^{\prime}$-GAGCTCTCACAACCCCATCTG-3' (reverse). The primers were based on the gene sequences from $\mathrm{NCBI}^{2}$, designed using Primer 5 software, and synthesized by Invitrogen (Shanghai). The PCR amplification products were inserted into the pMD19-T cloning vector (Takara, Dalian, China) and sequenced by Majorbio ${ }^{3}$. Secondary structure, tertiary structure,

\footnotetext{
${ }^{2}$ http://www.ncbi.nlm.nih.gov/

${ }^{3}$ www.majorbio.com
} 
typical domain of R2R3 MYB protein, motif, and phylogenetic tree analyses of the ZmMYB111 and ZmMYB148 proteins were predicted using online bioinformatics analysis and other software.

\section{Cloning Promoters of the PAL and 4CL Genes}

Promoters ( 2000 bp) of the PAL (GRMZM2G029048) and 4-coumaric acid COA ligase (4CL, GRMZM2G075333) were amplified from maize gDNA using PCR with the following primer pairs for PAL and 4CL, respectively: $5^{\prime}$ GAATTCAAACCATGCGTCAGTTGAC-3' (forward) and 5'ACGCGTTGCGGTTGCGACAC-3' (reverse), and 5'-GAATT CGACGAGTGATCAAAGGTT-3' (forward) and $5^{\prime}$-ACGC GTCTCAGACCTTTGCTC-3' (reverse). The PCR products were inserted into the pMD19-T cloning vector (Takara, Dalian, China) and sequenced by Majorbio ${ }^{4}$.

\section{Transcription Levels}

Quantitative real-time PCR (qRT-PCR) analysis was performed using SYBR Premix Ex TaqTM II (Tli RNaseH Plus; Clontech) according to the manufacturer's protocol on a CFX96TM RealTime PCR system (Bio-Rad, Hercules, CA, USA). The qRT-PCR reaction mixtures contained $0.5 \mu \mathrm{L}(0.4 \mu \mathrm{M})$ each primer, $1.0 \mu \mathrm{L}$

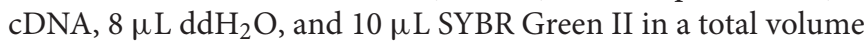
of $20 \mu \mathrm{L}$.

Primers for the three target genes (ZmMYB111, ZmMYB148, and $P A L$ ) were designed to amplify fragments of approximately $200 \mathrm{bp}$, and the maize actin gene (Actin) was used as a reference (Table 1). The qRT-PCRs were carried out in biological triplicates, and standard curves were verified with CFX Manager 3.1(Bio-Rad, USA) using a linear regression analysis of the threshold cycle CT value for each of 8 total DNA standard dilutions in each sample. The specificity of PCR primers was determined using melting curve analysis of the amplified products. The maize actin gene was used as the reference gene to normalize the expression of the target genes, which was calculated using the relative quantization method $\left(2^{-\Delta C t}\right)$.

\section{Subcellular Localization}

The GFP-ZmMYB111 and GFP-ZmMYB148 vectors were constructed to visualize subcellular localization.

${ }^{4}$ www.majorbio.com

TABLE 1| Real time PCR primer sequences for the three targeted genes and Actin.

\begin{tabular}{lcl}
\hline Gene name & JGI number & \multicolumn{1}{c}{ Primer sequence $\left(\mathbf{5}^{\prime} \mathbf{- 3} \mathbf{)}\right.$} \\
\hline ZmMYB111 & GRMZM2G104551 & F: GAACGACGACGCCGTGATAAG \\
& & R: TCTTCCAAAAGCCACTCACCAG \\
ZmMYB148 & GRMZM2G097636 & F: GCGGAGGGAAAGAGTGGTG \\
& & R: GCCCATTGGGTACTGGTAGTCC \\
PAL & GRMZM2G029048 & F: CGTCAACGACAACCCGCTCA \\
& & R: GAGGCCGTTGTTAGTAGTCGTTC \\
Actin & GRMZM2G320797 & F: TCACTACGACTGCCGAGCGAG \\
& & R: GAGCCACCACTGAGGACAACATTAC
\end{tabular}

The full-length cDNAs of ZmMYB111 and ZmMYB148 were amplified by PCR using the following primers: $5^{\prime}$ CGGGATCCATGGGTCGGCAGCCGT-3' (forward) and 5'-GC TCTAGACTAGAACTTTGCTCCGT-3' (reverse), and 5'-CGGG ATCCATGGGGAAGGGCC-3' (forward) and 5'-GCTCTA $\overline{\text { GA }}$ TCACAACCCCATCTG-3' (reverse). (The underlined sections in the forward and reverse primer sequences indicate BamHI and $\mathrm{XbaI}$ sites, respectively.) The PCR products were inserted into the pMD19-T cloning vector, and after digestion with $B a m H I$ and $\mathrm{XbaI}$, the resulting fragments were ligated into the pCAMBIA2300-35S-eGFP vector, which contained a GFP protein driven by the CaMV $35 \mathrm{~S}$ promoter (plasmid map in Supplementary Datasheet), amplified using PCR, sequenced, and named GFP-ZmMYB111 and GFP-ZmMYB148 after identification.

Onion epidermal cells were then bombarded with the GFPZma111 and GFP-Zma148 vectors, respectively, using a helium biolistic gun transformation system (Bio-Rad, USA), as described previously (Hu et al., 2012). The transformed cells were incubated on $1 / 2$ MS medium for $24-48 \mathrm{~h}$ at $28^{\circ} \mathrm{C}$. The subcellular localization of GFP fusion proteins was visualized using a BX61 fluorescence microscope with a wavelength of $488 \mathrm{~nm}$ (Olympus, Japan). Empty p2300-35S-eGFP vector was used as a control.

\section{Transcription Activation}

The pGBDKT7-ZmMYB111 and pGBDKT7-ZmMYB148 vectors were constructed for transcription activation analysis. The full-length cDNAs of ZmMYB111 and ZmMYB148 were amplified using PCR with the following primers: $5^{\prime}-$ CGGAATTCATGGGTCGGCAGCCGT-3' (forward) and 5'-CG GGATCCCTAGAACTTTGCTCCGT-3' (reverse), and 5'-CG GAATTCATGGGGAAGGGCC-3' (forward) and 5'-CGGGA TCCTCACAACCCCATCTG- $3^{\prime}$ (reverse). (The underlined sections in the forward and reverse primer sequences indicate EcoRI and BamHI sites, respectively.) The PCR products were inserted into the pMD19-T cloning vector, and after digestion with EcoRI and BamHI, the resulting fragments were ligated into the pGBKT7 DNA-binding domain vector (Clontech, USA), amplified using PCR, sequenced, and named PGBDKT7-ZmMYB111 and PGBDKT7-ZmMYB148 after identification.

Transcription activation analysis was performed in order to assess the presence of a protein activation domain (Fujita et al., 2004). For this analysis, both pGADT7-ZmaMYB111 and pGADT7-ZmaMYB148 vectors were transformed into AH109 yeast cells using the lithium acetate-mediated method, respectively (Gietz and Woods, 2002). The transformants were screened on $\mathrm{SD} /$-Trp medium, and positive clones were confirmed using PCR. The colonies were then screened on SD/Trp-His-Leu medium with X- $\alpha$-gal. In addition, the yeasts were further cultivated at $28^{\circ} \mathrm{C}$ for $3-5$ days to test transcription activation. The pGBDKT7-Lam and pGBDKT7-53 vectors and AH109 (no vector) were used as controls.

\section{Protein-DNA Interaction}

The yeast one-hybrid system is widely recognized as a valuable and straightforward technique for studying interactions between 
transcription factors and DNA. Accordingly, pGADT7ZmaMYB111 and pGADT7-ZmaMYB148 vectors were constructed for use in a yeast one-hybrid assay. The cDNAs of ZmMYB111 and ZmMYB148 were amplified using PCR with the following primers: 5'-CGGAATTCATGGGTCGGCAGCCGT-3' (forward) and 5' -CGAGCTCCTAGAACTTTGCTCCGT-3' (rev erse), and $5^{\prime}$-CGGAATTCATGGGGAAGGGCC-3' (forward) and $5^{\prime}$-CGAGCTCTCACAACCCCATCTG-3' (reverse). (The underlined sections in the forward and reverse primer sequences indicate EcoRI and SacI sites, respectively.) The PCR products were inserted into the pMD19-T cloning vector, and after digestion with EcoRI and SacI, the resulting fragments were ligated into the multiple cloning site (MCS) of the pGADT7Rec2 vector (Clontech, USA), amplified using PCR, sequenced, and named pGADT7-ZmaMYB111 and pGADT7-ZmaMYB148 after identification.

In addition, the promoters for PAL and 4CL were ligated into the EcoRI and MluI restriction sites, respectively, of the pHis2 plasmid (Clontech, USA), amplified using PCR, sequenced, and named pHis2-pPAL and pHis2-p4CL after identification. The related recombinant expression vectors were co-transformed into the Y187 yeast strain. Then, growth of the transformed yeast were compared on SD/-Trp/-Leu medium and SD/-Leu-UraHis + with $10 \mathrm{mM}$ 3-AT (3-amino-1,2,4-triazole) medium to test the expression of the His reporter gene. Empty pHis 2 was used as a negative control.

\section{Transient Expression Assays in Maize Endosperm}

The pBI221-ZmaMYB111 and pBI221-ZmaMYB148 vectors were constructed for transient expression analysis in maize endosperm. The cDNAs of ZmMYB111 and ZmMYB148 were amplified using PCR with the following primers: $5^{\prime}$ CCAAGCTTATGGGTCGGCAGCCGT-3' (forward) and $5^{\prime}$-CG GGATCCCTAGAACTTTGCTCCGT-3' (reverse), and 5' -CCAA GCTTATGGGGAAGGGCC-3' ${ }^{\prime}$ (forward) and $5^{\prime}$-CGGGATCC TCACAACCCCATCTG-3' (reverse). (The underlined sections in the forward and reverse primer sequences indicate HindIII and BamHI sites, respectively.) The PCR products were inserted into the pMD19-T cloning vector, and after digestion with HindIII and BamHI, the resulting fragments were ligated into the MCS of the pBI221 vector, in order to drive the LUC reporter gene (which replaced the GUS reporter gene), amplified using PCR, sequenced, and named pBI221-ZmaMYB111 and pBI221ZmaMYB148 after identification.

Meanwhile, the promoters of PAL and 4CL were ligated into the XbaI and SacI restriction sites, respectively, of the pBI221$\mathrm{ADH}$ plasmid, which contained the first intron of the AdhI gene in order to enhance promoter activity without altering promoter specificity, amplified with PCR, sequenced, and named pBI221-pPAL-ADH after identification. These were also used for transient expression.

Endosperm was isolated from immature (9 DAP) maize kernels that had been surface-sterilized with $75 \%$ ethanol and was placed in MS medium (Murashige and Skoog salts containing $1 \%$ agar and $10 \%$ sucrose) for $4 \mathrm{~h}$ prior to bombardment.
A Biolistic PDS-1000/He Particle Delivery System (Bio-Rad) was used to deliver gold particles coated with DNA. Each independent experiment consisted of four replicates, and was repeated 2-3 times with similar results. The bombarded endosperms were then cultivated for $36 \mathrm{~h}$ in order to analyze expression of the LUC reporter gene. Empty pBI221 vector, which contained the GUS gene driven by a maize ubiquitin promoter, was used as a control in order to correct for transfection efficiency. The fluorogenic assay for GUS activity was performed as previously described (Lu et al., 1998), and LUC activity was determined using a luciferase assay system (Promega, Madison, WI, USA). The fluorescence and luminescence were determined using a Luminoskan ${ }^{\mathrm{TM}}$ Ascent (Thermo, Rockford, IL, USA), as described in our previous report (Hu et al., 2012).

\section{RESULTS}

\section{Cloning and Bioinformatics Analysis}

The full-length cDNA sequences of ZmMYB111 and ZmMYB148 were amplified by RT-PCR, and the PCR products were analyzed using agarose gel electrophoresis. Sequencing revealed that the cDNA sequences of ZmMYB111 and ZmMYB148 were consistent with the GenBank report. The ZmMYB111 gene contains 828 bases and encodes a protein with 275 amino acids, an isoelectric point of 5.44, and a putative molecular weight of $30.05 \mathrm{kDa}$. In contrast, the ZmMYB148 gene contains 1149 bases and encodes a protein with 382 amino acids, an isoelectric point of 5.01, and a putative molecular weight of $40.65 \mathrm{kDa}$. Analysis using the PSIPRED software ${ }^{5}$ revealed that the n-termini of both proteins mainly consists of an $\alpha$-helix (Figure 1), including three $\alpha$-helix repeats that form a helix-turn-helix (HTH) motif as the DNA-binding domain, according to analysis using the SWISSMODEL software ${ }^{6}$ (Figure 2), and the HTH motif is the MYB transcription factor typical characteristics. In addition, through domain analysis using Expasy-Prosite ${ }^{7}$, we found that the ZmMYB111 and ZmMYB148 proteins included the conserved domain typical of R2R3-MYB transcription factors (Figure 3). Together, the above results confirmed that both ZmMYB111 and ZmMYB148 proteins belong to the MYB transcription factor family.

A phylogenetic (NJ) tree of the amino acid sequences of ZmMYB111, ZmMYB148, and other MYB transcription factors involved in phenylpropanoid metabolism was constructed using MAFFT ${ }^{8}$ and MEGA5.0 software (Figure 4). The results indicated that transcription factors with similar functions formed separate branches. Thus, ZmMYB111 may function similarly to AtMYB85 (i.e., in the regulation of lignin synthesis), since they were on the same branch, and alternatively, ZmMYB148 may function similarly to $\mathrm{Zm} 1$ (i.e., in anthocyanin biosynthesis), since they were on the same branch. Conserved motif analysis of ZmMYB111, ZmMYB148, and the other MYB transcription

\footnotetext{
${ }^{5}$ http://bioinf.cs.ucl.ac.uk/psipred/

${ }^{6} \mathrm{http}: / /$ swissmodel.expasy.org

${ }^{7}$ http://prosite.expasy.org/

${ }^{8} \mathrm{http}: / /$ mafft.cbrc.jp/alignment/server/index.html
} 


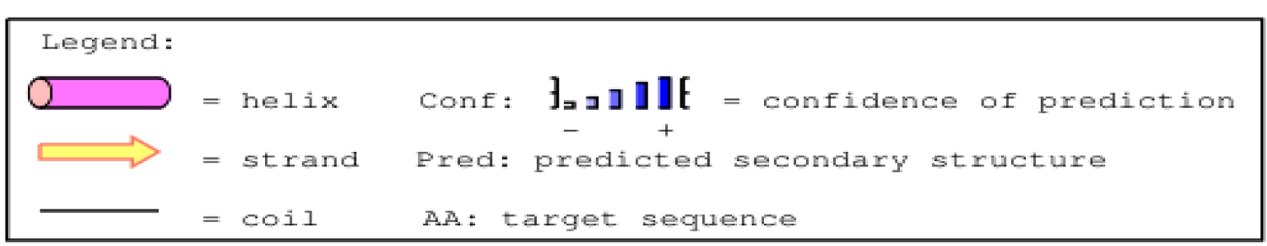

\section{Conf : 101}

Pred:

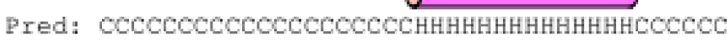

AA; MGRGRAPCCAKVGLNKGSWTPEEDMRI I Y IOKYGHANWF

$\begin{array}{llll}10 & 20 & 30 & 40\end{array}$

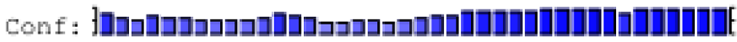

Pred: $\square-\sigma$

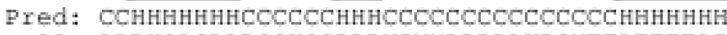

AA: ALPKOAGLLROGKSCRLFWINYLFEDLRAGNFTAEEEAI $\begin{array}{llll}50 & 60 & 70 & 80\end{array}$

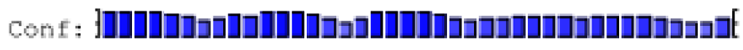

Pred: $\longrightarrow-$

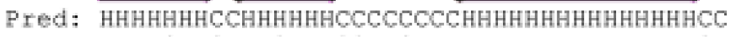

RA: IKLHGLLGNKWSKIASCLPGRTDNE IKNVWNTHLKKRVSE $\begin{array}{llll}90 & 100 & 110 & 120\end{array}$

\section{Conf: | ||}

Pred:

Pred: cocecococococecococcoccecoccococcococcoc

AA: AGEERGAAGSKKKKKKKT TKAAAGGGAEAPLPLP SPSPSS $\begin{array}{llll}130 & 140 & 150 & 160\end{array}$

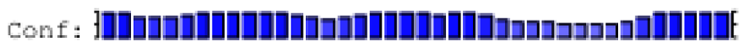

Pred:

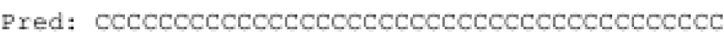

AA: STTTTNESSGDSGEOQSNMMSKEADDELDLENFEMHEMLD

$$
\begin{array}{llll}
170 & 180 & 190 & 200
\end{array}
$$

\section{Conf: $\mid$ |}

Pred:

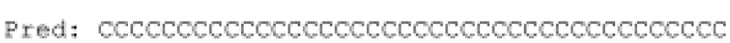

AA: VDDESFGFGTLVDTAPAPYGSAVSVSASAATSPCASSTSE

$$
\begin{array}{llll}
210 & 220 & 230 & 240
\end{array}
$$

\section{Conf : |}

Pred:

Pred: ceccococococococcococcocococococococcec

AA: PPASAPPGVDDLLVLPEIDMGHELWSI IDGDAREAPAPRC

$\begin{array}{llll}250 & 260 & 270 & 280\end{array}$

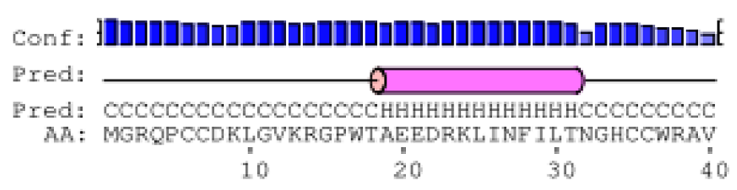

Conf : h|

Pred: 0

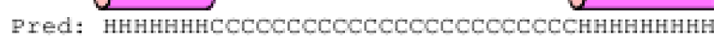

AA: PKLAGLLRCGKSCFLRWTNLRPDLKEGLLTDAEEOVVID

$$
\begin{array}{llll}
50 & 60 & 70 & 80
\end{array}
$$

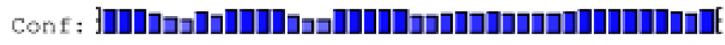

Pred:

A.A : LHAKLGNRWSKIA.AKLE GRTDNE I KNHWNT I I KKKLI I KMG

$90 \quad 100 \quad 110 \quad 120$

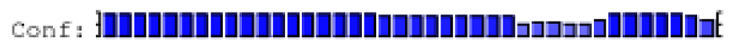

Pred:

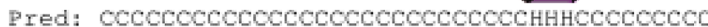

AA: IDPVTHEPLDRFT TSSGPATTSQSTKSDEATKEQSPQNDD

$$
\begin{array}{llll}
130 & 140 & 150 & 160
\end{array}
$$

conf :

Pred: 0

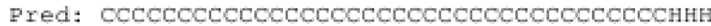
AA: AVIRDVPADGCSPTESSTNTVSTGGSSSSGGGGHDQDPLV

$\begin{array}{llll}170 & 180 & 190 & 200\end{array}$

Conf: \} \}

Pred:

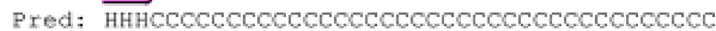

AA: KWLLEEEPATGDEAWLNFTGSVDVDEESSIAAGPELLPWD

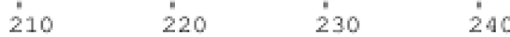

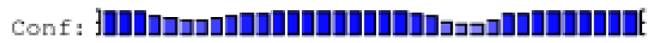

Pred:

Pred: occecoccecoceccoccecoccecoccecoccec

$A A$ : GATDWLLDYQDFGLGDSSLVDGYMVNNNSSNGAKE

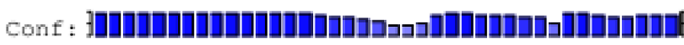

Fred: 0

Pred: CCCCCCCCCCCCCCCCCCCCCCHHHHHHHHHHCCCCCCCO AA: QRNEAEPTNGADAGSHGAEGKEWWLEDLERELGLWGTVED

$$
\begin{array}{llll}
290 & 300 & 310 & 320
\end{array}
$$

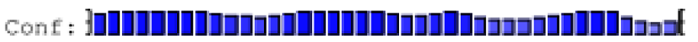

Pred:

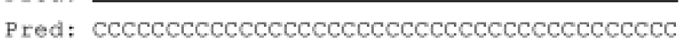

AA: YQYEMGPOGLLVADHPDP LPAMVDQPVSCYFQAGPASAVL,

$$
330 \quad 340 \quad 350 \quad 360
$$

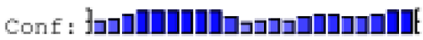

Pred:

Pred: coccoccococeEeccocococ

AA : QELFGYPVEATAVTGSINQMGL

$$
370 \quad 380
$$

FIGURE 1 | Secondary structures of the amino acid sequences of ZmMYB111 (left) and ZmMYB148 (right), as predicted by PSIPRED analysis (http:// bioinf.cs.ucl.ac.uk/psipred/). 


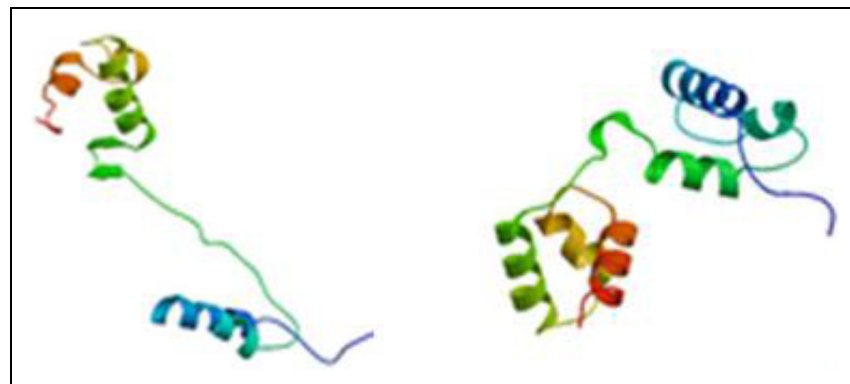

FIGURE 2 | Tertiary structure of ZmMYB111 (left) and ZmMYB148 (right), as predicted by SWISS-MODEL analysis (http://swissmodel. expasy.org/).

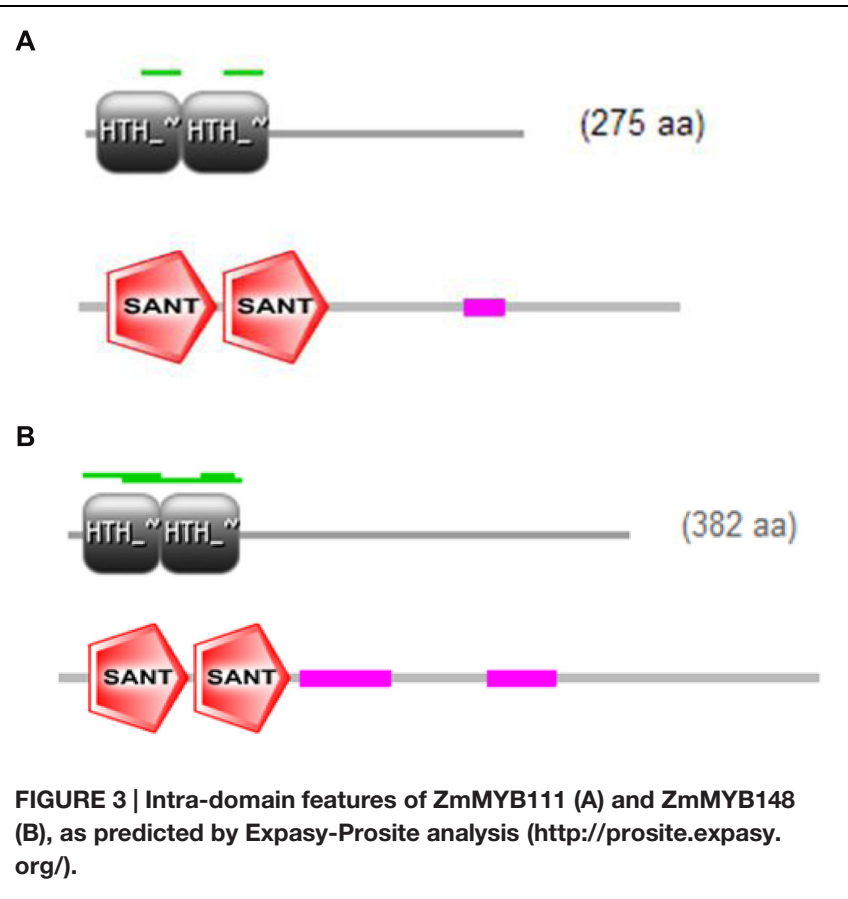

factors using the MEME software ${ }^{9}$ showed that both ZmMYB111 and ZmMYB148 shared three motifs (motif 1, motif 2, and motif 3 ) in common with all 21 other MYB domain transcription factors analyzed in this paper (Figure 5). In addition, ZmMYB111 also contained motif 4, which is found in AtMYB32, Zm42, $\mathrm{Zm31}$, and AtMYB85, all of which are involved in lignin synthesis; motif 5, which is found in ZmMYB-IF35, ZmMYBIF25, AtMYB/PAP1, and AtMYB/PAP2, all of which are involved in flavonoid synthesis; and motif 7 , which is found in $\mathrm{ZmC1}$, PL2, AtMYB/PAP1, and AtMYB/PAP2, all of which are involved in anthocyanin synthesis (Cone et al., 1993; Lesnick and Chandler, 1998). Of the seven conserved motifs found in ZmMYB148, three (motif 7, motif 10, and motif 12) are also found in $\mathrm{Zm1}$, which is involved in flavonoid biosynthesis, and another (motif 11) is also found in AtMYB32, Zm42, $\mathrm{Zm31}$, and AtMYB63, all of which are involved in lignin

${ }^{9}$ http://meme.nbcr.net/meme/ biosynthesis (Figure 5; Franken et al., 1994; Heine et al., 2007).

\section{Expression Pattern Analyses}

When we examined the expression patterns of ZmMYB111 and ZmMYB148 in different organs of maize plants, we found that ZmMYB111 was expressed in roots, endosperm, seeds, and stems. Furthermore, it was expressed most highly in lower stems and was extremely low in leaves, seedling roots, or male flowers (Figure 6A). In contrast, the expression of ZmMYB148 was highest in endosperm, followed by roots and lower stems, and was extremely low in leaves, seedling roots, pith, and female flowers (Figure 6B). These results suggest that the ZmMYB111 and ZmMYB148 genes might play an important role in maize endosperm development.

The PAL gene is a key component of phenylpropanoid metabolism, and it may be the target gene of the transcription factor ZmMYB148 (Du et al., 2012); therefore, its expression pattern was also investigated. Our results indicated that the PAL gene was transcribed in maize endosperm (Figure 6C). Therefore, we concluded that it could be useful for conducting transient expression assays in maize endosperm in order to measure the regulation functions of $\mathrm{ZmMYB111}$ and ZmMYB148.

\section{Subcellular Localization}

Most transcription factors localized to the nucleus. The subcellular localization of ZmMYB111 and ZmMYB148 proteins was determined in onion epidermal cells. As shown in Figure 7, both ZmMYB111 and ZmMYB148 were located in the nucleus, whereas the control GFP was localized in both cytoplasm and the nucleus.

\section{Transcription Activation}

The constructs of pGBKT7-ZmMYB111 and pGBKT7ZmMYB148 were separately transformed into yeast AH109 cells and screened on SD/-Trp medium. Positive clones were identified using PCR and were further cultivated on SD/-Trp/His/10mmol3-AT/X- $\alpha$-gal mediums. We found that the yeast cells transformed with pGBKT7-ZmMYB111 and pGBKT7ZmMYB148 were able to grow and change the color of the medium to blue, which suggests that both ZmMYB111 and ZmMYB148 possess transcription activation activities (Figure 8).

\section{Protein-DNA Interaction}

Phenylalanine ammonia-lyase and 4CL are the key enzymes in phenylpropanoid metabolism. Promoters of the PAL and 4CL genes were amplified from maize B73 gDNA using PCR and analyzed by agarose gel electrophoresis. Sequencing results revealed that the promoter sequences were consistent with GenBank report, and the lengths of the promoters were 1867 and $1873 \mathrm{bp}$, respectively. The promoters were separately ligated into the pHis2 plasmid to generate the pHis2-pPAL and pHis2-p4CL constructs that will be used for yeast one-hybrid screening for DNA-protein interactions.

The constructs were co-transformed into the yeast strain Y187, and the yeast cells were screened using 


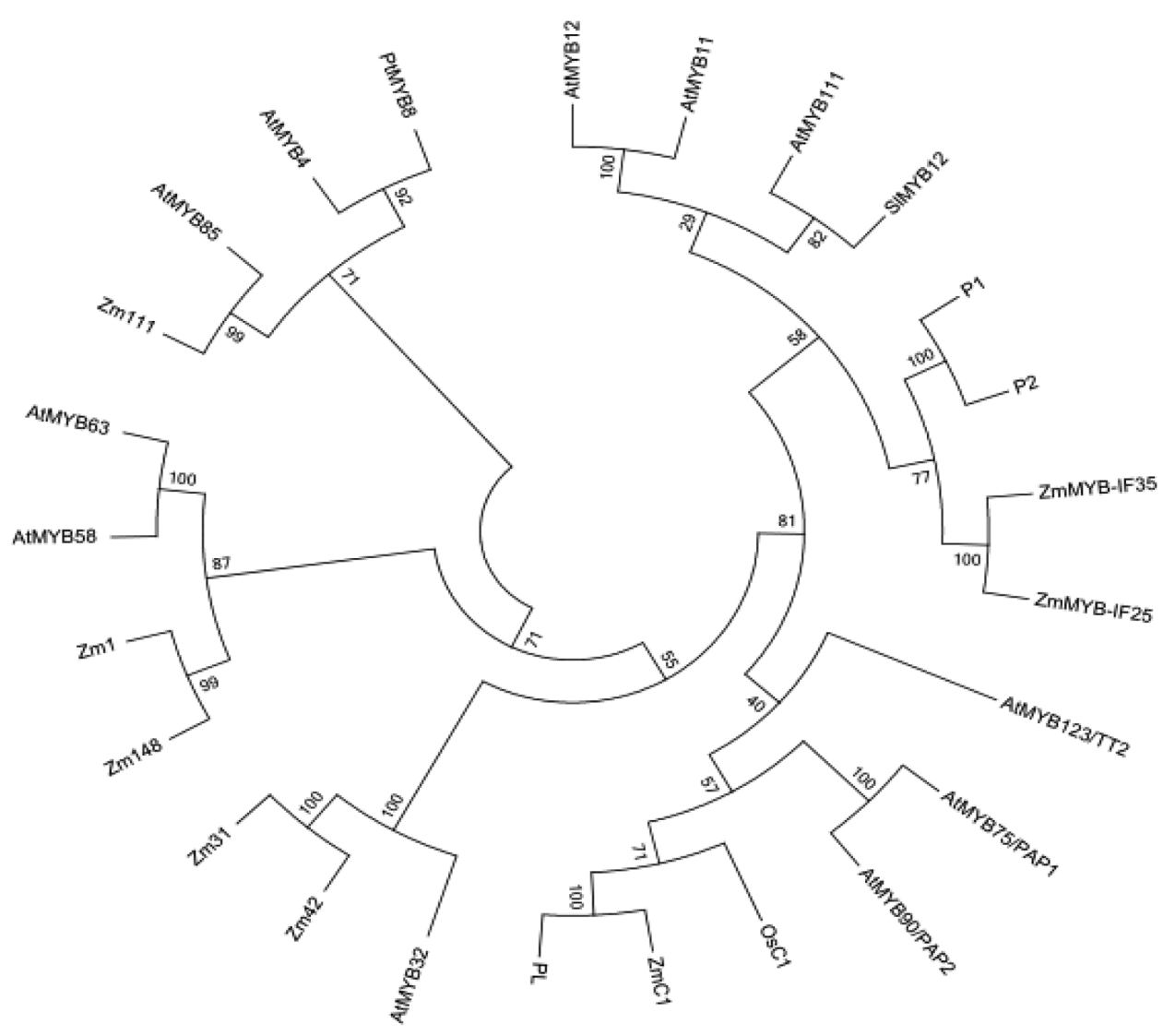

FIGURE 4 | Phylogenetic tree of ZmMYB111 and ZmMYB148 with other MYB transcription factors, based on amino acid sequences and built using MAFFT (http://mafft.cbrc.jp/alignment/server/index.html) and MEGA5.0 software. The tree was generated using the neighbor-joining method with bootstrap support by 1000 replicates.

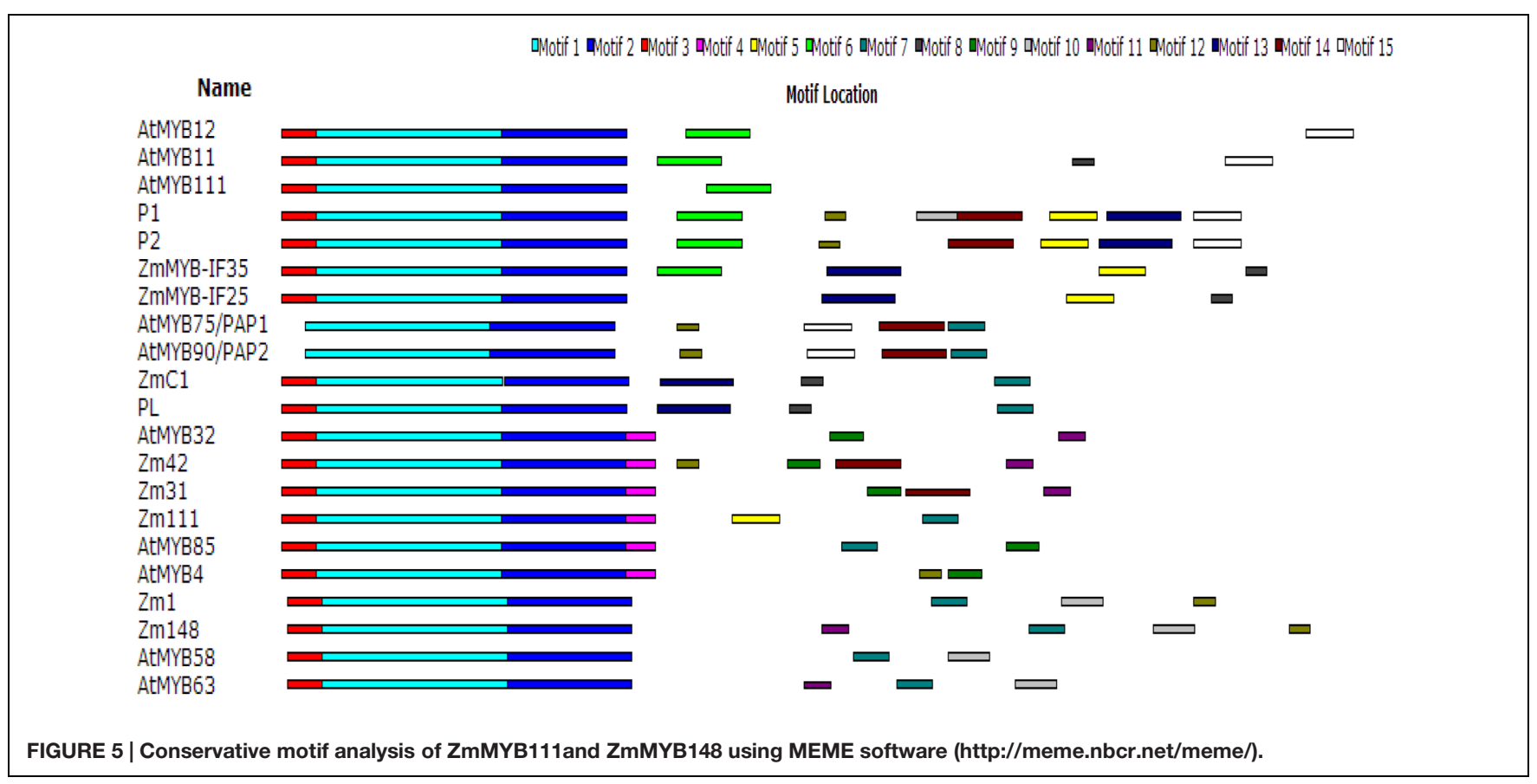




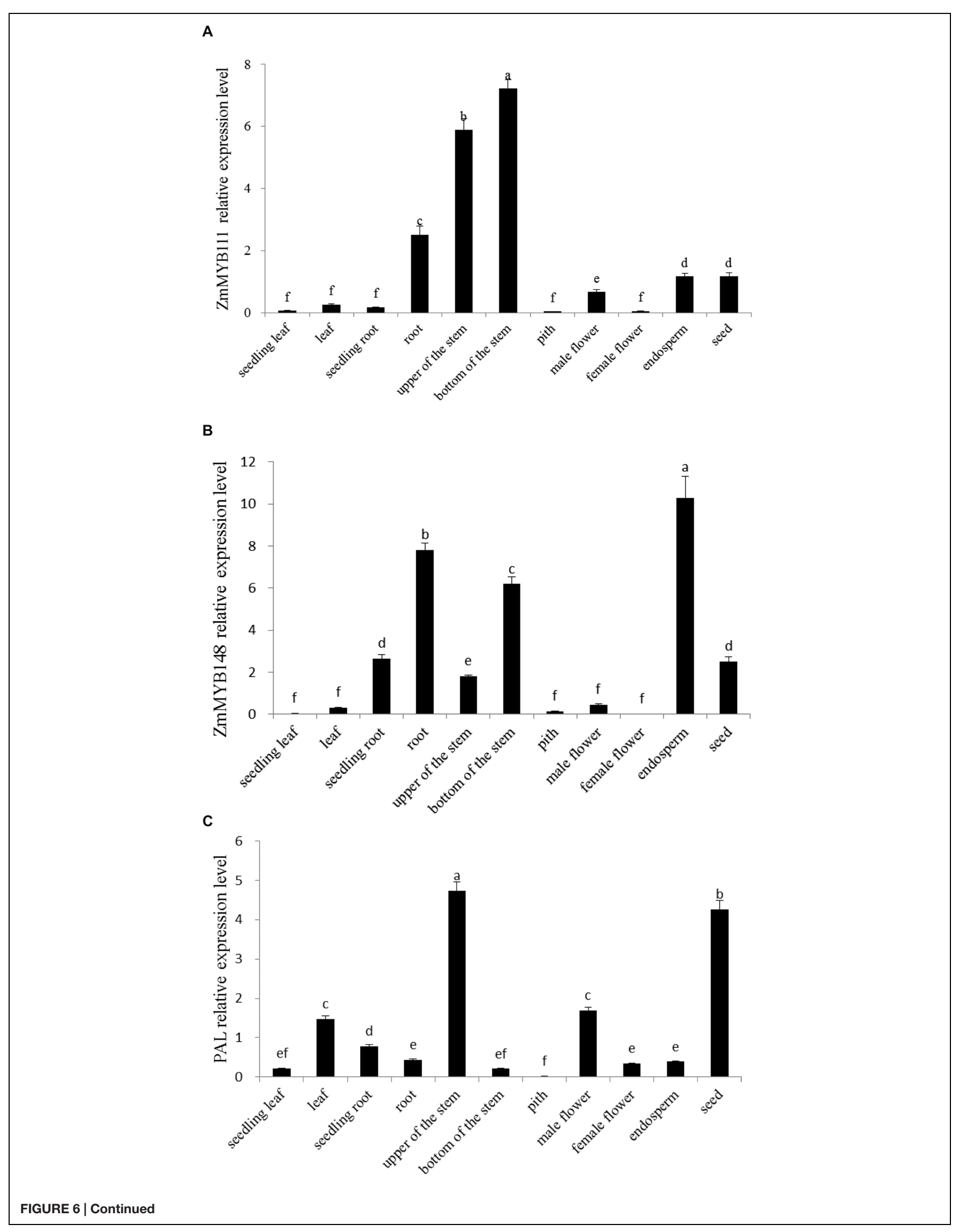




\section{FIGURE 6 | Continued}

Expression analysis of ZmMYB111 (A), ZmMYB148 (B), and PAL (C) genes in different organs of maize. Relative expression levels were measured using qRT-PCR. Data represent the mean \pm SD of three biological replicate experiments and three technical replicates and were analyzed by Duncan's test ( $n=3$ ). Different lower case letter (a-f) indicates the significant difference at $P \leq 0.05$.

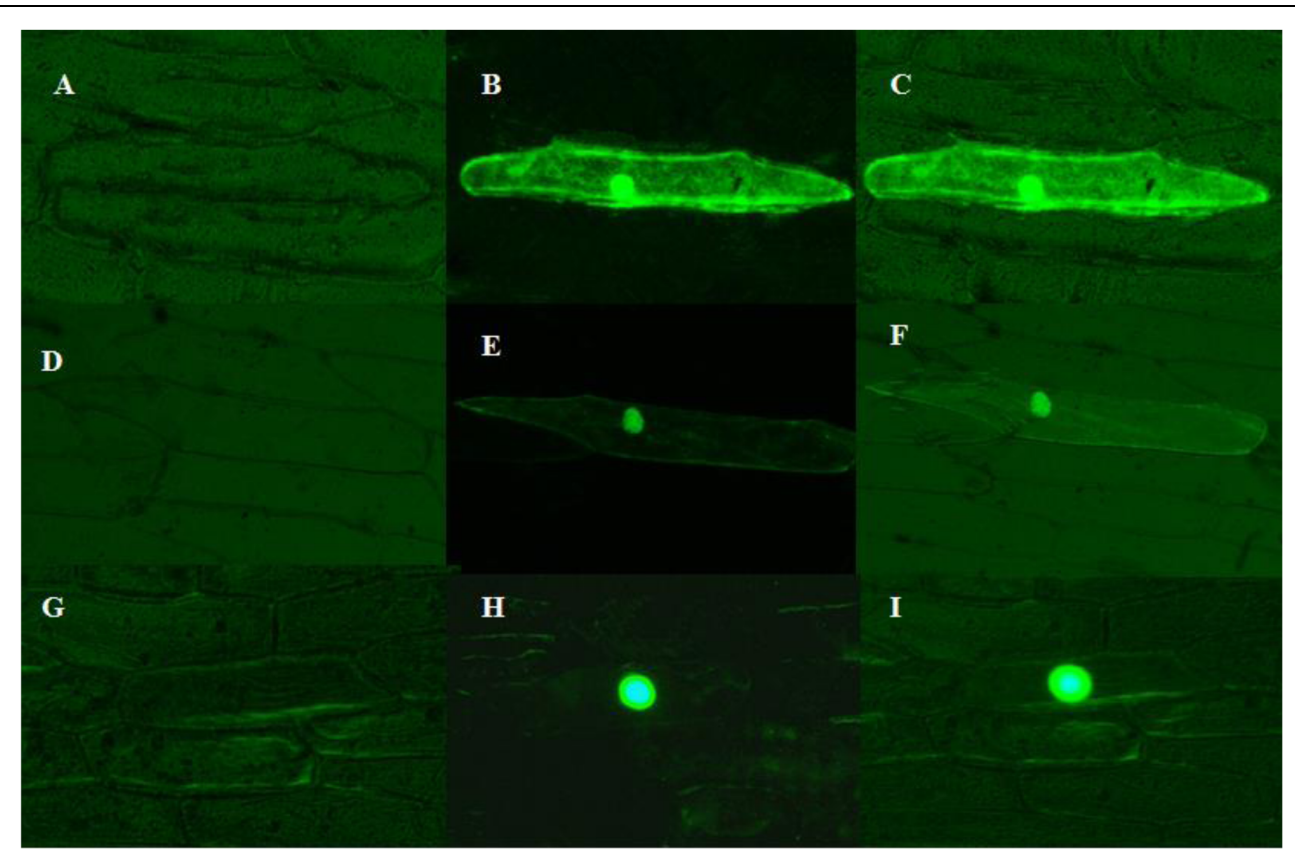

FIGURE 7 | Subcellular localization of ZmMYB111 and ZmMYB148. The fusion protein, driven by the 35S promoter, was transiently expressed in onion epidermal cells and analyzed by fluorescent microscopy. GFP alone was used as a control. (A) p2300-35S-eGFP image under white light. (B) p2300-35S-eGFP image under blue light. (C) p2300-35S-eGFP image under blue light and white light. (D) eGFP- ZmMYB111 image under white light. (E) eGFP- ZmMYB111 image under blue light. (F) eGFP- ZmMYB111 image under blue light and white light. (G) eGFP- ZmMYB148 image under white light. (H) eGFP- ZmMYB148 image under blue light. (I) eGFP- ZmMYB148 image under blue light and white light.
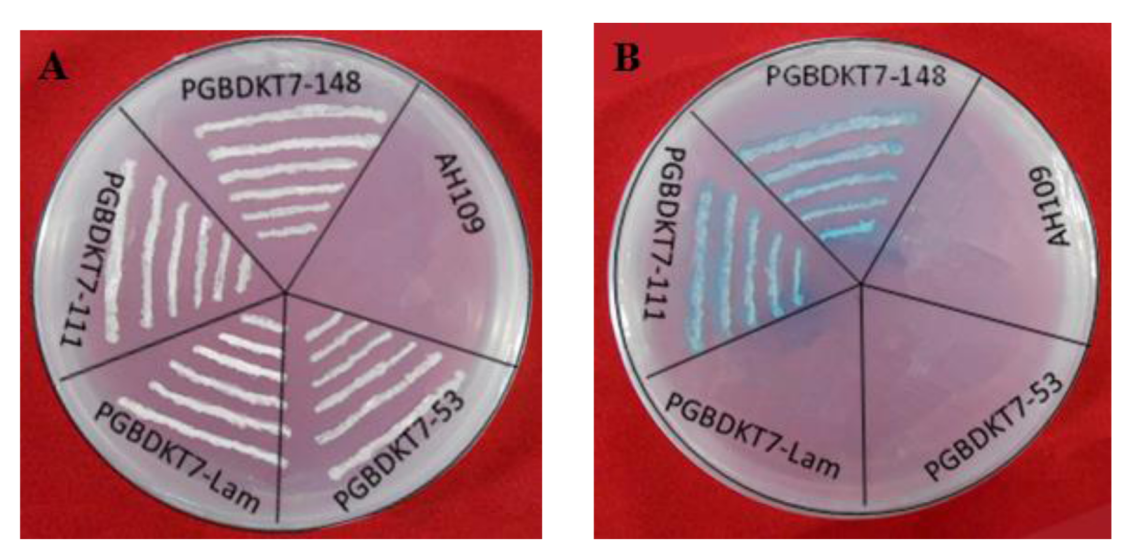

FIGURE 8 | Transactivation activity assays of ZmMYB148 and ZmMYB111 in yeast. AH109, yeast strain AH109 without any vector; PGBDKT7-Lam and PGBDKT7-53, negative controls. The transformants were screened on SD/-Trp medium (A). The colonies were screened on SD/-Trp-His-Leu medium with $\mathrm{X}-\alpha-\mathrm{gal}(\mathbf{B})$.

a synthetic dropout nutrient medium. As is shown in Figure 9, yeast transformants that contained the plasmid pGADT7-ZmaMYB111/pGADT7-ZmaMYB148 and pHis2pPAL or pGADT7-ZmaMYB111/pGADT7-ZmaMYB148 and
pHis2-p4CL were able to grow on the SD/-Trp/-Leu and $\mathrm{SD} /$-Trp/-Leu/-His/100mmol3-AT mediums. However, the yeast transformants harboring pHis2 could only grow on the $\mathrm{SD} /$-Trp/-Leu medium (Figure 9). These results suggest that 

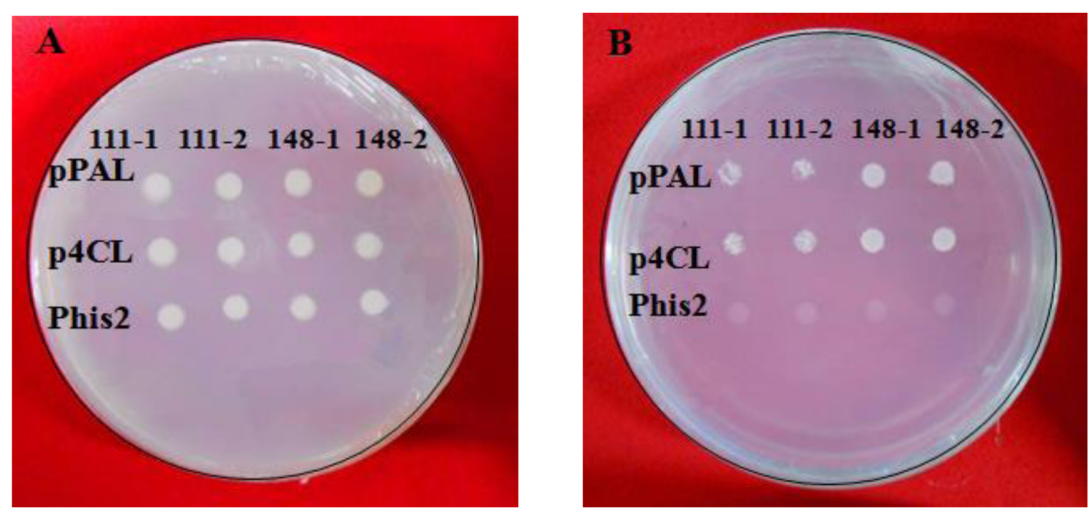

FIGURE 9 | Binding characteristics of ZmMYB111 and ZmMYB148 transcription factors with the promoter of PAL and 4CL using yeast one-hybrid analysis. The related recombinant expression vectors were co-transformed into the yeast strain $\mathrm{Y} 187$, and empty pHis2 was used as a negative control. (A) The growth status of the transformed yeasts on SD/-Trp/-Leu medium. (B) The growth status of the transformed yeasts on SD/-Leu-Ura-His+ medium with 10 mM 3-AT.

both ZmaMYB111 and ZmMYB148 are able to bind to the promoters of the PAL and $4 \mathrm{CL}$ genes and may regulate their transcription.

\section{Transient Expression Assays in Maize Endosperm}

The relative constructs were co-transformed into maize endosperms in order to analyze the binding characteristics of ZmaMYB111 and ZmMYB148 transcription factors to the PAL promoter. As shown in Figure 10, the activity ratio of LUC/GUS was only 0.5 in the case of the pBI221-pPAL$\mathrm{ADH}$. However, in the presence of pBI221-ZmaMYB111 and pBI221-ZmaMYB148, the ratio of LUC/GUS reached 1.6 and 4.8 and increased by 3 and 8 times, respectively (Figure 10). The results indicate that the ZmMYB111 and ZmMYB148 transcription factors are able to bind to the PAL promoter and improve the expression of the PAL gene.

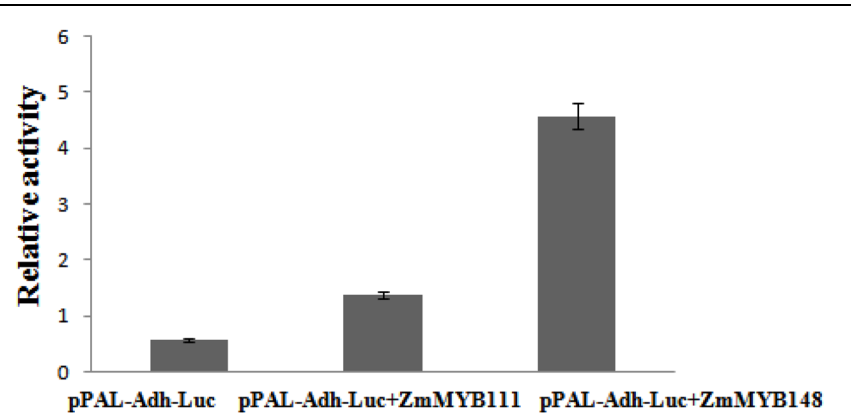

FIGURE 10 | Phenylalanine ammonia-lyase (PAL) promoter activity, induced by the transcription factors ZmMYB111 (middle) and ZmMYB148 (right) in our transient expression experiment. The related constructs were co-transformed into 9 DAP endosperm, and empty pBI221 was used as a control (left) in order to correct for transfection efficiency. The activities of GUS and LUC were measured, and the activity ratio of LUC/GUS was calculated to reflect the relative activity of the PAL promoter.

\section{DISCUSSION}

Phenylpropanoid metabolism plays an important role in crop resistance to abiotic and biotic stressors, as well as in quality (Richard and Paiva, 1995; Payyavula et al., 2012). The process begins when $\mathrm{L}$-phenylalanine is transformed to $t$-cinnamic acid by PAL, the activity of which is regulated at the transcriptional level, depending on the stage of cell differentiation and exposure to various kinds of stress (Weitzel and Petersen, 2010). In addition, PAL is an important branch point enzyme that links primary and secondary plant metabolism, including the production of anthocyanins, flavonoids, monolignols, coumarins, etc. (Weitzel and Petersen, 2010). In this paper, the ZmMYB111 and ZmMYB148 transcription factors demonstrated clear regulatory influence on the transcription of the PAL gene, so it is possible that they are also involved in regulating the synthesis of phenylpropanoid compounds.

Indeed, a number of MYB transcription factors have been confirmed in the regulation of flavonoid and lignin biosynthesis in Arabidopsis. For example, the transcription factors MYB58, MYB46, MYB63, MYB75, and MYB83 have all been shown to be involved in the regulation of lignin synthesis (Zhou et al., 2009; Bhargava et al., 2010; Ko et al., 2014), and MYB12, MYB75, MYB/PAP1, and AtMYB/PAP2 are known to be involved in flavonoid synthesis (Cone et al., 1993; Lesnick and Chandler, 1998; Nakabayashi et al., 2014). Furthermore, in maize, the MYB31 and MYB42 transcription factors were shown to inhibit lignin synthesis (Sonbol et al., 2009; Fornalé et al., 2010), ZmC1 was shown to regulate anthocyanin synthesis (Franken et al., 1994), and MYB-IF35 was shown to be involved in flavonoid synthesis (Heine et al., 2007).

In general, fewer MYB transcription factors have been described as involved in phenylpropanoid metabolism in maize, and even those have mainly been involved in the regulation of downstream reactions. In contrast, our study suggests that the ZmMYB111 and ZmMYB148 transcription factors might regulate the first key enzyme gene (the PAL gene) simultaneously, and ZmMYB111 and ZmMYB148 might be involved in the 
synthesis of both flavonoids and lignins. Accordingly, we speculated that the ZmMYB111 and ZmMYB148 transcription factors could have a much broader application in maize breeding.

In our study, RT-qPCR analysis indicated that the expressions of ZmMYB111 and ZmMYB148 in leaves, roots, and stems were higher in mature plants than in seedlings. In other words, their expression levels were higher in mature tissues than in young tissues of the same plants (Figures 6A,B). Interestingly, it was previously reported that R2R3-MYB transcription factors involved in lignin synthesis were mainly expressed in lignified organization, and higher expression levels have been found in mature tissues than in tender tissues (McCarthy et al., 2011; Handakumbura and Hazen, 2012), which is consistent with our results. Bioinformatics analysis also revealed that ZmMYB111 and ZmMYB148 were associated with lignin synthesis. Thus, we speculate that the ZmMYB111 and ZmMYB148 genes might play a pivotal role in the lignin synthesis of maize.

Expression of the PAL gene has been reported at various levels in all organs of plants (Fock-Bastide et al., 2014; de Jong et al., 2015), and our results also indicated that the PAL gene could be transcribed in all organs of maize, including endosperm, and especially in stems and seeds (Figure 6C). Production of stable, fertile transgenic lines is an essential technique in gene function studies; however, transformation in maize is difficult with low transformation rate and long breeding times. Therefore, using transient transformation approaches to study gene function is an alternative method (Reyes et al., 2010; Wu et al., 2014). Because the ZmMYB148 gene was highly expressed in maize endosperm, 9 DAP endosperm was used as the receptor of transient expression, which was conducted in order to determine the regulatory effect of the ZmMYB148 protein on the expression of the PAL gene. Transient expression in maize endosperms is a good method for identifying the roles of target genes expressed in endosperm and has been used successfully in our previous studies (Hu et al., 2012; Zhang et al., 2014). In addition, flavonoids and their derivatives are abundant in mature maize kernels, and

\section{REFERENCES}

Bhargava, A., Mansfield, S. D., Hall, H. C., Douglas, C. J., and Ellis, B. E. (2010). MYB75 functions in regulation of secondary cell wall formation in the Arabidopsis inflorescence stem. Plant Physiol. 154, 1428-1438. doi: 10.1104/pp.110.162735

Boerjan, W., Ralph, J., and Baucher, M. (2003). Lignin biosynthesis. Annu. Rev. Plant Biol. 54, 519-546. doi: 10.1146/annurev.arplant.54.031902.13 4938

Bohnert, H. J., Nelson, D. E., and Jensen, R. G. (1995). Adaptations to environmental stresses. Plant Cell 7, 1099-1111. doi: 10.1105/tpc.7. 7.1099

Cai, H., Tian, S., and Dong, H. (2012). Large scale in silico identification of MYB family genes from wheat expressed sequence tags. Mol. Biotechnol. 52, 184-192. doi: 10.1007/s12033-011-9486-3

Cone, K. C., Cocciolone, S. M., Burrm, F. A., and Burr, B. (1993). Maize anthocyanin regulatory gene $\mathrm{pl}$ is a duplicate of $\mathrm{cl}$ that functions in the plant. Plant Cell 5, 1795-1805. doi: 10.1105/tpc.5.12.1795

de Jong, F., Hanley, S. J., Beale, M. H., and Karp, A. (2015). Characterisation of the willow phenylalanine ammonia-lyase (PAL) gene family reveals expression differences compared with poplar. Phytochemistry 117, 90-97. doi: 10.1016/j.phytochem.2015.06.005 they have specified the functions (Koes et al., 2005; Falcone Ferreyra et al., 2012; Wen et al., 2014). In conclusion, we speculate that the ZmMYB148 gene might play a role in the synthesis of flavonoids, based on the results of conserved motifs analysis, the phylogenetic tree, and qPCR. Therefore, the gene may find an application in breeding programs for the development of maize with improved flavonoid content.

\section{AUTHOR CONTRIBUTIONS}

YH designed and supervised this study. JZ, SZ, Hui Li, Yangping $\mathrm{Li}$, and $\mathrm{HH}$ performed the experiments. YH, Hanmei Liu, and GY performed the data analysis. Yinghong Liu performed the fieldwork. JZ designed the experiments and was extensively involved in the writing the manuscript.

\section{FUNDING}

This work was supported by the National Key Basic Research Program of China (No: 2014CB138200), and the National Natural Science Foundation of China (No: 31571682).

\section{ACKNOWLEDGMENTS}

We thank Mr. Zhi Huang for technical support. This work was partly supported by the Maize Research Institute, Sichuan Agricultural University.

\section{SUPPLEMENTARY MATERIAL}

The Supplementary Material for this article can be found online at: http://journal.frontiersin.org/article/10.3389/fpls.2016.00148

Du, H., Feng, B. R., Yang, S. S., Huang, Y. B., and Tang, Y. X. (2012). The R2R3-MYB transcription factor gene family in maize. PLoS ONE 7:e37463. doi: 10.1371/journal.pone.0037463

Dubos, C., Stracke, R., Grotewold, E., Weisshaar, B., Martin, C., and Lepiniec, L. (2010). MYB transcription factors in Arabidopsis. Trends Plant Sci. 15, 573-581. doi: $10.1016 /$ j.tplants

Falcone Ferreyra, M. L., Rius, S. P., and Casati, P. (2012). Flavonoids: biosynthesis, biological functions, and biotechnological applications. Front. Plant Sci. 3:222. doi: 10.3389/fpls.2012.00222

Fock-Bastide, I., Palama, T. L., Bory, S., Lécolier, A., Noirot, M., and Joët, T. (2014). Expression profiles of key phenylpropanoid genes during Vanilla planifolia pod development reveal a positive correlation between PAL gene expression and vanillin biosynthesis. Plant Physiol. Biochem. 74, 304-314. doi: 10.1016/j.plaphy.2013.11.026

Fornalé, S., Shi, X., Chai, C., Encina, A., Irar, S., Capellades, M., et al. (2010). ZmMYB31 directly represses maize lignin genes and redirects the phenylpropanoid metabolic flux. Plant J. 64, 633-644. doi: 10.1111/j.13 65-313X

Franken, P., Schrell, S., Peterson, P. A., Saedler, H., and Wienand, U. (1994). Molecular analysis of protein domain function encoded by the mybhomologous maize genes $\mathrm{C} 1, \mathrm{Zm} 1$ and $\mathrm{Zm} \mathrm{38}$. Plant J. 6, 21-30. doi: 10.1046/j.1365-313X.1994.6010021.x 
Fraser, C. M., and Chapple, C. (2011). The phenylpropanoid pathway in Arabidopsis. Arabidopsis Book 9:e0152. doi: 10.1199/tab.0152

Fujita, M., Fujita, Y., Maruyama, K., Seki, M., Hiratsu, K., Ohme-Takagi, M., et al. (2004). A dehydration-induced NAC protein, RD26, is involved in a novel ABA-dependent stress-signaling pathway. Plant J. 39, 863-876. doi: 10.1111/j.1365-313X.2004.02171.x

Fulton, T. M., Chunwongse, J., and Tanksley, S. D. (1995). Microprep protocol for extraction of DNA from tomato and other herbaceous plants. Plant Mol. Biol. Rep. 13, 207-209. doi: 10.1007/BF02670897

Gietz, R. D., and Woods, R. A. (2002). Transformation of yeast by lithium acetate/single-stranded carrier DNA/polyethylene glycol method. Methods Enzymol. 350, 87-96. doi: 10.1016/S0076-6879(02)50957-5

Handakumbura, P. P., and Hazen, S. P. (2012). Transcriptional regulation of grass secondary cell wall biosynthesis: playing catch-up with Arabidopsis thaliana. Front. Plant Sci. 3:74. doi: 10.3389/fpls.2012.00074

Heine, G. F., Malik, V., Dias, A. P., and Grotewold, E. (2007). Expression and molecular characterization of ZmMYB-IF35 and related R2R3-MYB transcription factors. Mol. Biotechnol. 37, 155-164. doi: 10.1007/s12033-0070061-X

Hu, Y. F., Li, Y. P., Zhang, J. J., Liu, H., Tian, M. L., and Huang, Y. B. (2012). Binding of ABI4 to a CACCG motif mediates the ABA-induced expression of the ZmSSI gene in maize (Zea mays L.) endosperm. J. Exp. Bot. 63, 5979-5989. doi: $10.1093 /$ jxb/ers 246

Kanei-Ishii, C., Sarai, A., Sawazaki, T., Nakagoshi, H., He, D. N., and Ogata, K. (1990). The tryptophan cluster: a hypothetical structure of the DNA-binding domain of the myb protooncogene product. J. Biol. Chem. 265, 19990-19995.

Katiyar, A., Smita, S., Lenka, S. K., Rajwanshi, R., Chinnusamy, V., and Bansal, K. C. (2012). Genome-wide classification and expression analysis of MYB transcription factor families in rice and Arabidopsis. BMC Genomics 13:544. doi: 10.1186/1471-2164-13-544

Ko, J. H., Jeon, H. W., Kim, W. C., Kim, J. Y., and Han, K. H. (2014). The MYB46/MYB83-mediated transcriptional regulatory programme is a gatekeeper of secondary wall biosynthesis. Ann. Bot. 114, 1099-1107. doi: $10.1093 / \mathrm{aob} / \mathrm{mcu} 126$

Koes, R., Verweij, W., and Quattrocchio, F. (2005). Flavonoids: a colorful model for the regulation and evolution of biochemical pathways. Trends Plant Sci. 10, 236-242. doi: 10.1016/j.tplants.2005.03.002

Lesnick, M. L., and Chandler, V. L. (1998). Activation of the maize anthocyanin gene $\mathrm{a} 2$ is mediated by an element conserved in many anthocyanin promoters. Plant Physiol. 117, 437-445. doi: 10.1104/pp.117.2.437

Lu, C. A., Lim, E. K., and Yu, S. M. (1998). Sugar response sequence in the promoter of a rice alpha-amylase gene serves as a transcriptional enhancer. J. Biol. Chem. 273, 10120-10131. doi: 10.1074/jbc.273.17.10120

Mansfield, S. D. (2009). Solutions for dissolution-engineering cell walls for deconstruction. Curr. Opin. Biotechnol. 20, 286-294. doi: 10.1016/j.copbio.2009.05.001

McCarthy, R. L., Zhong, R., and Ye, Z. H. (2011). Secondary wall NAC binding element (SNBE), a key cis-acting element required for target gene activation by secondary wall NAC master switches. Plant Signal. Behav. 6, 1282-1285. doi: 10.4161/psb.6.9.16402

Moura, J. C. M. S., Bonine, C. A. V., Viana, J., Dornelas, M. C., and Mazzafera, P. (2010). Abiotic and biotic stresses and changes in the lignin content and composition in plants. J. Integr. Plant Biol. 52, 360-376. doi: 10.1111/j.17447909.2010.00892.x

Nakabayashi, R., Yonekura-Sakakibara, K., Urano, K., Suzuki, M., Yamada, Y., Nishizawa, T., et al. (2014). Enhancement of oxidative and drought tolerance in Arabidopsis by overaccumulation of antioxidant flavonoids. Plant J. 77, 367-379. doi: 10.1111/tpj. 12388

Payyavula, R. S., Navarre, D. A., Kuhl, J. C., Pantoja, A., and Pillai, S. S. (2012). Differential effects of environment on potato phenylpropanoid and carotenoid expression. BMC Plant Biol. 12:39. doi: 10.1186/14712229-12-39
Petrussa, E., Braidot, E., Zancani, M., Peresson, C., Bertolini, A., Patui, S., et al. (2013). Plant Flavonoids-biosynthesis, transport and involvement in stress responses. Int. J. Mol. Sci. 14, 14950-14973. doi: 10.3390/ijms1407 14950

Ramakrishna, A., and Ravishankar, G. A. (2011). Influence of abiotic stress signals on secondary metabolites in plants. Plant Signal. Behav. 6, 1720-1731. doi: 10.4161/psb.6.11.17613

Reyes, F. C., Sun, B., Guo, H., Gruis, D. F., and Otegui, M. S. (2010). Agrobacterium tumefaciens-mediated transformation of maize endosperm as a tool to study endosperm cell biology. Plant Physiol. 153, 624-631. doi: 10.1104/pp.110.154930

Richard, A. D., and Paiva, N. L. (1995). Stress-induced phenylpropanoid metabolism. Plant Cell 7, 1085-1097. doi: 10.2307/3870059

Solecka, D. (1997). Role of phenylpropanoid compounds in plant responses to different stress factors. Acta Physiol. Plant. 19, 257-268. doi: 10.1007/s11738997-0001-1

Sonbol, F. M., Fornalé, S., Capellades, M., Encina, A., Touriño, S., Torres, J. L., et al. (2009). The maize ZmMYB42 represses the phenylpropanoid pathway and affects the cell wall structure, composition and degradability in Arabidopsis thaliana. Plant Mol. Biol. 70, 283-296. doi: 10.1007/s11103-009-9473-2

Vanholme, R., Demedts, B., Morreel, K., Ralph, J., and Boerjan, W. (2010). Lignin biosynthesis and structure. Plant Physiol. 153, 895-905. doi: 10.1104/pp.110.155119

Vogt, T. (2010). Phenylpropanoid biosynthesis. Mol. Plant 3, 2-20. doi: $10.1093 / \mathrm{mp} / \mathrm{ssp} 106$

Wang, C., Shi, X., Liu, L., Li, H., Jetty, S. S., David, A., et al. (2013). Genomic resources for gene discovery, functional genome annotation, and evolutionary studies of maize and its close relatives. Genetics 195, 723-737. doi: 10.1534/genetics.113.157115

Weitzel, C., and Petersen, M. (2010). Enzymes of phenylpropanoid metabolism in the important medicinal plant Melissa officinalis L. Planta 232, 731-742. doi: 10.1007/s00425-010-1206-X

Wen, W., Li, D., Li, X., Gao, Y., Li, W., Li, H., et al. (2014). Metabolome-based genome-wide association study of maize kernel leads to novel biochemical insights. Nat. Commun. 5, 34-38. doi: 10.1038/ncomms4438

Wu, H. Y., Liu, K. H., Wang, Y. C., Wu, J. F., Chiu, W. L., Chen, C. Y., et al. (2014). AGROBEST: an efficient Agrobacterium-mediated transient expression method for versatile gene function analyses in Arabidopsis seedlings. Plant Methods 10:19. doi: 10.1186/1746-4811-10-19

Zhang, J. J., Chen, J., Yi, Q., Hu, Y. F., Liu, H. M., Liu, Y. H., et al. (2014). Novel role of ZmaNAC36 in co-expression of starch synthetic genes in maize endosperm. Plant Mol. Biol. 84, 359-369. doi: 10.1007/s11103-013-0153-x

Zhou, J. L., Lee, C. H., Zhong, R. Q., and Ye, Z. H. (2009). MYB58 and MYB63 are transcriptional activators of the lignin biosynthetic pathway during secondary cell wall formation in Arabidopsis. Plant Cell 21, 248-266. doi: $10.1105 /$ tpc. 108.063321

Conflict of Interest Statement: The authors declare that the research was conducted in the absence of any commercial or financial relationships that could be construed as a potential conflict of interest.

The reviewer AM and handling Editor declared their shared affiliation, and the handling Editor states that the process nevertheless met the standards of a fair and objective review.

Copyright ( 2016 Zhang, Zhang, Li, Du, Huang, Li, Hu, Liu, Liu, Yu and Huang. This is an open-access article distributed under the terms of the Creative Commons Attribution License (CC BY). The use, distribution or reproduction in other forums is permitted, provided the original author(s) or licensor are credited and that the original publication in this journal is cited, in accordance with accepted academic practice. No use, distribution or reproduction is permitted which does not comply with these terms. 